\title{
EFECTO CICATRIZANTE DEL CHAMPÚ LÍQUIDO DE Colletia spinosissima J. GMELIN “TACSANA” EN RATONES
}

\author{
Wound healing effect of liquid shampoo of Colletia spinosissima J. Gmelin "tacsana" in mice
}

\author{
Beatriz Herrera ${ }^{1}$, Jorge L. Arroyo ${ }^{2}$, Oscar Herrera ${ }^{3}$, Martín Condorhuamán ${ }^{4}$, Bertha Pari ${ }^{3}$, Edith Loyola ${ }^{3}$ \\ ${ }^{1}$ Unidad de Posgrado, Facultad de Farmacia y Bioquímica, UNMSM. ${ }^{2}$ Facultad de Medicina Humana, UNMSM. ${ }^{3}$ Facultad \\ de Farmacia y Bioquímica, Universidad Nacional San Luis Gonzaga de Ica. ${ }^{4}$ Facultad de Farmacia y Bioquímica, UNMSM.
}

\section{RESUMEN}

El objetivo del estudio fue determinar el efecto cicatrizante del champú a base de Colletia spinosissima (tacsana) en lesiones inducidas en piel del ratón. Se utilizaron 36 ratones albinos, de peso entre 30-35 g, a los que se depiló en la zona dorsal, aplicándose lidocaína gel al $4 \%$ en la zona depilada para inducir lesiones (heridas) en la piel. Los animales fueron asignados en cinco grupos, cada uno constituido por seis ratones. El diseño experimental estuvo conformado de la siguiente manera: G1: Control negativo; G2: Control positivo; G3, G4 y G5: formulado al 10\%; 20\%; y 30\%, respectivamente; G6: Pantene ${ }^{\circledast}$. Se administró el champú líquido dos veces al día, por catorce días consecutivos. En los resultados se observó en los grupos 4 y 5 disminución de la lesión sobre la piel al cuarto día, los animales del grupo 5 presentaron un proceso de costras y reparación de la piel, con formación de colágeno en el estudio histológico. Se concluye que el champú líquido a base de Colletia spinosissima (tacsana) al 30\% presentó efecto cicatrizante en lesiones inducidas en piel de ratón.

Palabras clave: Cicatrización, Colletia spinosissima, champú líquido.

\section{SUMMARY}

The objective of the study was to determine the healing effect of Colletia spinosissima (tacsana) shampoo based on induced injuries in mouse skin. Were used 36 albino mice, weighing between $30-35 \mathrm{~g}$; to which was depilated in the dorsal area, applying lidocaine gel $4 \%$ in the depilated area to induce lesions (wounds) on the skin. The animals were allocated into five groups, each group consisting of six mice. The experimental design was composed as follows: G1: negative control, G2: positive control; G3, G4 and $G_{5}$ formulated 10\%; $20 \%$; and 30\%, respectively; G6: Pantene ${ }^{\oplus}$. The liquid shampoo was administered twice daily for fourteen consecutive days. Results shows that in groups 4 and 5 was reduced the injury to the skin on the fourth day, the animals of group 5 exhibited a process of scabs and skin repair, with collagen formation in the histological study. It is concluded that liquid based shampoo Colletia spinosissima (tacsana) provided $30 \%$ healing effect in mouse skin induced injuries.

Keywords: Wound healing, Colletia spinosissima, liquid shampoo.

\section{INTRODUCCIÓN}

as heridas crónicas representan uno de los principales problemas médicos y económicos (1). La investigación básica ha permitido la comprensión de la estimulación e inhibición de la curación de una herida y han provisto la base para la introducción de nuevos métodos de tratamiento ${ }^{(2)}$. La cicatrización conduce a la restauración del tejido e integridad y ocurre a través de un proceso altamente organizado, que pese a ser continuo, puede dividirse arbitrariamente en cuatro fases principales: hemostasia, inflamación, proliferación, y la remodelación. Estas fases corresponden a un sistema biológico molecular complejo que requiere la proliferación celular, angiogénesis que están reguladas por factores de crecimiento ${ }^{(3)}$.

El colágeno es una de las principales proteínas de la matriz extracelular y es uno de los componentes que últimamente contribuyen a la cicatrización de las heridas. Los taninos promocionan la cicatrización de heridas a través de varios mecanismos celulares, que incluyen la quelación de los radicales libres y de las especies reactivas oxigenadas promoviendo la contracción de la herida, incrementando la creación de nuevos vasos sanguíneos (angiogénesis) y la formación de fibroblastos ${ }^{(4)}$.

Colletia spinosissima J. Gmelin, conocidamente como "tacsana", es una especie vegetal muy conocida por los pobladores de la serranía peruana de Huancavelica por ser útil para lavados corporales y ayudar a la cicatrización de heridas cutáneas ${ }^{(5)}$.

Dentro de los metabolitos secundarios principales reportados en las especies naturales con posible efecto cicatrizante están las saponinas, flavonoides, triterpenos, taninos y alcaloides ${ }^{(6)}$. Los compuestos fenólicos tales 
Tabla 1. Escala de valoración macroscópica a los diferentes grupos experimentales para la evaluación del efecto cicatrizante.

\begin{tabular}{ccc}
\hline Respuesta & Aspecto morfológico & Puntuación \\
\hline NINGUNA & $\begin{array}{c}\text { Piel rojiza, caliente y con signos de } \\
\text { inflamación leve y/o moderada. } \\
\text { REGULAR }\end{array}$ & $\begin{array}{c}\text { Se podía ver cambio de color de la } \\
\text { piel (disminución). }\end{array}$ \\
BUENA & $\begin{array}{c}\text { Mejoría de la inflamación, y } \\
\text { desaparición de las lesiones } \\
\text { superficiales. }\end{array}$ & 3 \\
MUY BUENA & $\begin{array}{c}\text { Proceso de cicatrización muy } \\
\text { notorio mejoramiento de la } \\
\text { epidermis y recuperación de la piel. }\end{array}$ & 4 \\
\hline
\end{tabular}

Tabla 2. Prueba preliminar cualitativa de los metabolitos secundarios del extracto etanólico de Colletia spinosissima (tacsana).

\begin{tabular}{lcc}
\hline \multicolumn{1}{c}{ Reactivo } & Resultado & Metabolitos \\
\hline Ninhidrina & $(+)$ & Aminoácidos Libres \\
Gelatina & $(+++)$ & Taninos \\
Cloruro Férrico $\left(\mathrm{Fecl}_{3}\right)$ & $(+++)$ & Compuestos Fenólicos \\
Dragendörff & $(+)$ & Alcaloides \\
Mayer & $(+)$ & Alcaloides \\
Shinoda & $(+++)$ & Flavonoides \\
Prueba de la Espuma & $(+++)$ & Saponinas \\
\hline
\end{tabular}

$(-)$ = Ausencia; $(+)=$ Poca cantidad; $(++)=$ Regular cantidad; $(+++)=$ abundante cantidad .

comolosácidos fenólicos, flavonoides, y taninos juegan un rol importante en la cicatrización de heridas, los taninos actúan sobre los radicales libres, triterpenos y flavonoides promueven la cicatrización debido a sus propiedades astringentes y antimicrobianas y las saponinas, por sus propiedades antioxidantes y antimicrobianas, parecen ser responsables de la contracción de la herida y elevar la tasa de reepitelización ${ }^{(7)}$.

Muchas plantas medicinales son útiles para el tratamiento de enfermedades de la piel como Alternanthera sessilis, Morinda citrifolia, Lycopodium

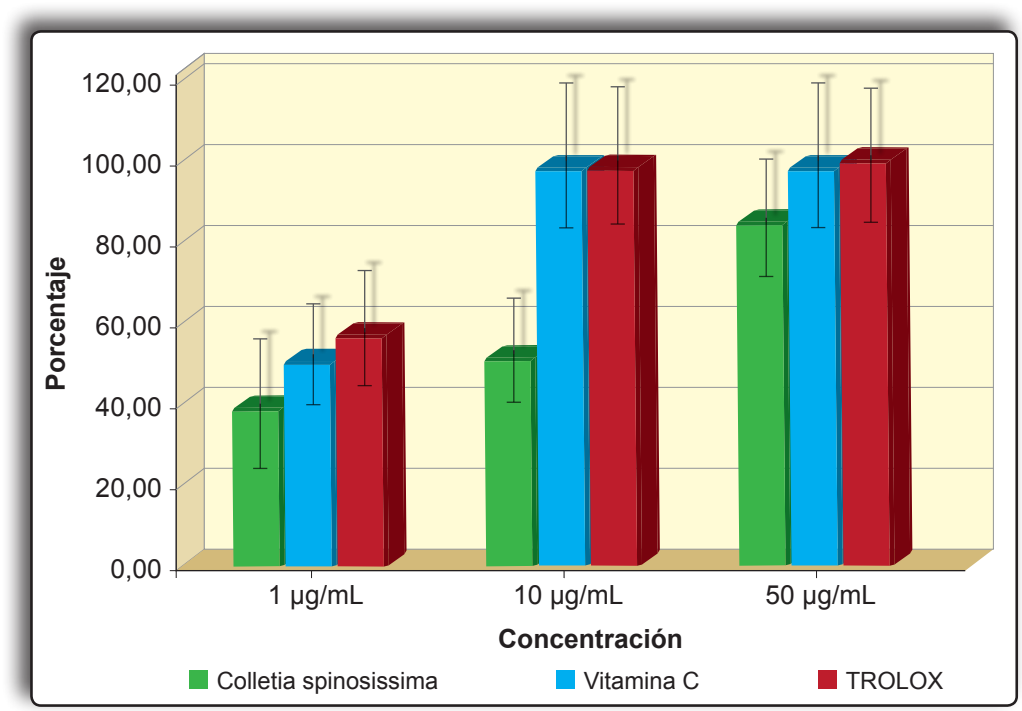

Figura 1. Porcentaje de captación del radical DPPH del extracto etanólico de Colletia spinosissima. serratum, Sesamum indicum, Catharanthus roseus, Cecropia peltata, Euphorbia hirta, Ginkgo biloba, Clerodendrum serratum, Pterocarpus santalinus, Lawsonia alba, Napoleonaea imperialis, Kaempferia galanga, Paeonia officinalis, Prosopis cineraria y Trigonella foenum-graecum ${ }^{(8)}$.

La presente investigación tuvo por objetivo evaluar el efecto cicatrizante del champú líquido de Colletia spinosissima J. Gmelin "tacsana" en lesiones inducidas en piel de ratón.

\section{MATERIAL Y MÉTODOS}

La investigación es de tipo experimental, prospectiva y longitudinal. La especie vegetal fue colectada en el distrito de Manta - Tasta, provincia de Huancavelica, en el departamento de Huancavelica, a 3500 metros de altitud, realizándose su clasificación taxonómica en el Museo de Historia Natural de la Universidad Nacional Mayor de San Marcos, según la siguiente posición sistemática de acuerdo a Cronquist (1988):

División : Magnoliophyta

Clase : Magnoliopsida

Orden : Rhamnales

Familia : Rhamnaceae

Género : Colletia

Especie : Colletia spinosissima J. Gmelin

Nombre vulgar : "tacsana"

El estudioexperimental serealizóen el Laboratorio de Farmacología de la Facultad de Medicina Humana de la Universidad Nacional Mayor de San Marcos.

La obtención del extracto se realizó por el método de maceración alcohólica de $1 \mathrm{~kg}$ de corteza y brote tiernos con tres litros de etanol 96\% durante siete días con agitación diaria. Posteriormente, se filtró y evaporó a sequedad, en estufa de aire circulante, a una temperatura menor de $40^{\circ} \mathrm{C}$, obteniéndose un peso $25 \mathrm{~g}$ de extracto seco. El análisis cualitativo de los metabolitos secundarios se realizó con los respectivos reactivos de identificación, calificándose como ausencia y presencia si hay cambio de coloración y/o precipitación ${ }^{(9)}$.

\section{Determinación de la actividad antioxidante}

La actividad antioxidante in vitro se realizó mediante el método de neutralización 
del radical 1, 1-difenil-2-picrilhidrazilo (DPPH) ${ }^{(10)}$, para lo cual se preparó solución metanólica de DPPH o,o1 mM; solución metanólica de Colletia spinosissima 1,0; 10,0 y 50,o $\mu \mathrm{g} / \mathrm{mL}$; y patrón de referencia (Trolox y vitamina $\mathrm{C}$ ). Las muestras y los controles reaccionaron con el reactivo DPPH, realizándose la lectura a $517 \mathrm{ym}$ luego de 30 minutos de reacción.

\section{Elaboración del champú líquido}

Para el estudio fue necesario emplear la base de un champú comercial ${ }^{(11)}$ y el champú liquido del extracto de Colletia spinosissima.

Champú base: Agua destilada, metilglucosamida (espesante) y Bromidox (conservante).

Champú control: Pantene ${ }^{\circledR}$, contenido $12 \mathrm{~mL}$.

Preparación del champú experimental: La base de $70 \mathrm{~mL}$ se fraccionó en 3 frascos, c/u con 23,3 mL, luego se añadió el extracto etanólico de Colletia spinosissima con un pH 6,5 en diferentes concentraciones.

Frasco 1: Champú a base de C. spinosissima al 10\%.

Frasco 2: Champú a base de $C$. spinosissima al 20\%.

Frasco 3: Champú a base de $C$. spinosissima al 30\%.

Evaluación in vivo de la cicatrización de las heridas provocadas por irritación inducida

La investigación farmacológica para la evaluación del efecto cicatrizante ${ }^{(11)}$, se realizó en 36 ratones albinos cepa Balb/C 53, machos, de $30 \mathrm{~g}$ aproximadamente de peso corporal, obtenidos en el Bioterio del Instituto Nacional de Salud - Centro Nacional de Productos Biológicos - Chorrillos. Los cuales fueron distribuidos en 6 grupos de 6 animales cada uno, a los que se depiló la superficie dorsal con Opilca ${ }^{\circledR}$ (sales del ácido tioglicolico), luego se aplicó anestesia con lidocaína en gel al $4 \%$ (vía tópica) y se procedió a inducir la irritación con una cinta adhesiva en la superficie ventral del ratón. Al primer grupo se aplicó o,1 $\mathrm{mL}$ de extracto del frasco 1 al 10\%; al segundo grupo $0,3 \mathrm{~mL}$ del frasco 2 al $20 \%$; tercer grupo 0,4 $\mathrm{mL}$ del frasco 3 al 30\%; al cuarto grupo $0,2 \mathrm{~mL}$ del champú comparador (Pantene ${ }^{\circledast}$ ); al quinto grupo o,2 $\mathrm{mL}$ de base; $\mathrm{y}$ al sexto grupo no se aplicó tratamiento. Los ratones recibieron el preparado dos veces al día durante un periodo de 14 días. La respuesta al efecto cicatrizante, desde el punto de vista macroscópico, fue evaluada según el criterio descrito en la tabla 1.

\section{Análisis de datos}

Para el análisis estadítico de los resultados, se utilizó el programa Statistical Package for the Social
Sciences (SPSS) versión 15.0. Los resultados fueron evaluados con estadística descriptiva en tablas y figuras.

\section{RESULTADOS}

En la tabla 2 se observa la presencia de metabolitos, principalmente saponinas, taninos y flavonoides.

En el ensayo de actividad antioxidante se logró comparar la actividad antioxidante del extracto etanólico con el Trolox y vitamina $\mathrm{C}$, siendo estos últimos superiores al extracto (figura 1).

En el estudio anatomopatológico se observa que el formulado al 30\% fue efectivo al completar el proceso de cicatrización y restaurar el tejido dérmico dañado (figura 2).

En la escala de valoración macroscópica aplicada a los diferentes grupos experimentales para la evaluación del efecto cicatrizante se observa, en la figura 3, que el formulado al 30\% presento una respuesta muy buena.

\section{DISCUSIÓN}

La irritación inducida a la piel altera la composición y la arquitectura de la matriz extracelular dérmica, causando inflamación, enrojecimiento en la piel ${ }^{(13)}$. En la presente investigación se demostró que el champú de Colletia spinosissima presenta efecto cicatrizante en lesiones inducidas en piel de ratón, favoreciendo la reparación celular.

El estudio microscópico y macroscópico realizado en la piel de los ratones, mostró efecto (queratinización) a nivel de las células córneas (corniocitos que están compuestos mayormente por queratina), siendo el efecto cicatrizante mayor con el champú liquido de Colletia spinosissima al 30\%, debido a su contenido de saponinas, triterpenoides, flavonoides y taninos determinados en el screening fitoquímico preliminar que se realizó al extracto etanólico ${ }^{(14,15)}$. De acuerdo a las observaciones macroscópicas se pudo apreciar que los animales tratados con el formulado al 10\% de C. spinosissima formaban una costra muy gruesa que no se dispersaba bien en la zona aplicada y lejos de beneficiar la cicatrización impedía el buen desarrollo de la misma. Esto se asoció con una baja dispersión y penetración del formulado 1 en los tejidos, lo que limitó su acción de reepitelización y cicatrización de las heridas. Estas observaciones correspondieron con las obtenidas en los estudios histológicos, reflejándose en los estadíos alcanzados para cada tratamiento (figura 2). 


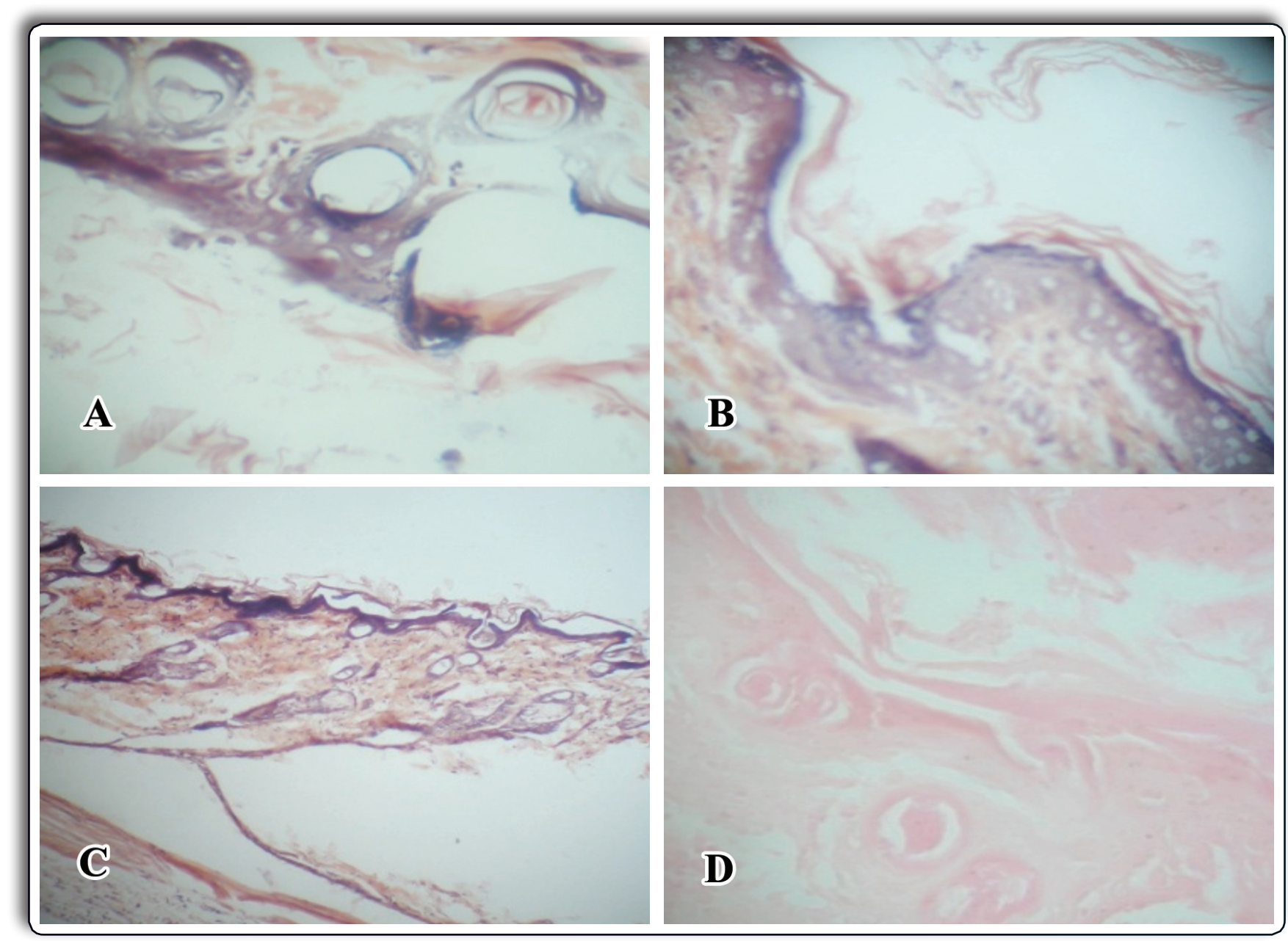

Figura 2. Microfotografía de piel de ratón en el estudio experimental del efecto cicatrizante sobre lesiones dérmicas inducidas. 400X. A. Control de lesión: piel con hiperqueratosis superficial, debajo de capa cornea existen células mononucleares y linfocitos (inflamación); lesión (+++) cicatrización (0). B. Formulado 10\%: capa superficial con adelgazamiento de la epidermis, incluso ampollas intradérmicas y en áreas hay pérdida de epidermis por abrasión; lesión (+) cicatrización (+). C. Formulado 20\%: la queratina es discontinua en toda la superficie, como si hubiera emulsionado; hay separación de las capas que conforman la epidermis, formando vesículas o flictenas; lesión (+), cicatrización (+). D. Formulado 30\%: empastamiento de epidermis; presencia de colágeno (cicatrización inicial); lesión (+) cicatrización (+).

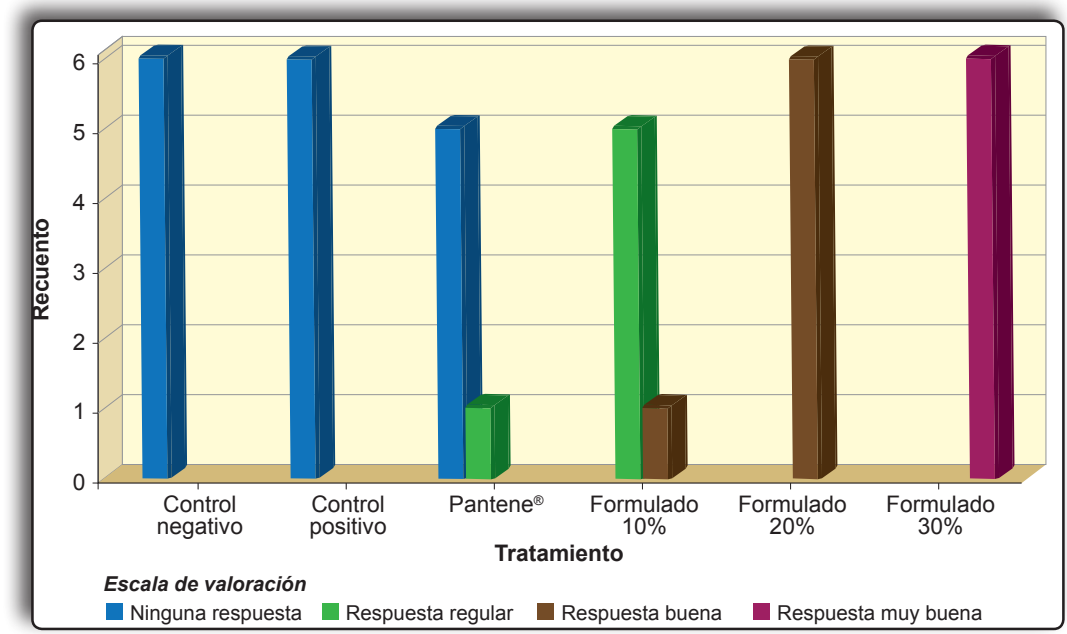

Figura 3. Valoración macroscópica de la cicatrización al día 14 post inducción de lesión dérmica en piel de ratón.
Los radicales libres y los productos de reacción oxidativa producen daño en los tejidos, particularmente en desórdenes del conectivo como la fibrosis; por tanto, debe tenerse en cuenta que el tejido cicatrizal no es ajeno a ello. De ahí que la eliminación de las especies reactivas oxigenadas podría ser una estrategia en la reparación de heridas ${ }^{(8)}$. El extracto etanólico de Colletia spinosissima presenta muybuena actividad antioxidante al neutralizar el radical DPPH a una concentración de $50 \mu \mathrm{g} / \mathrm{mL}$ con un $83 \%$ de inhibición (figura 1), siendo menor a los estándares conocidos como el trolox y vitamina C. Estos hallazgos justificarían el efecto cicatrizante producido sobre las 
lesiones inducidas en piel y su capacidad de proteger de las complicaciones que se puedan generar en el periodo de reepitelización.

Los animales cuyas evaluaciones alcanzaron la escala 3 y 4 de cicatrización fueron considerados como criterio de positividad en cuanto a calidad de regeneración del tejido (contenido de fibras colágenas, vasos de neoformación y presencia de folículos pilosos), lo que permitió demostrar que el efecto cicatrizante es dosis dependiente (figura 3).

Estos resultados mostraron una gran diferencia con relación a los controles en las características de la regeneración y formación de nuevo tejido, alcanzando alguno de los individuos la estructura total de piel normal en el período de observación al último día de experimentación. Los formulados de champú líquido a concentraciones de 20 y $30 \%$ fueron capaces de estimular la cicatrización tanto cuantitativa como cualitativamente, al producir una epitelización rápida y con escala 4 en la maduración de la dermis. La formación de colágeno alcanzada en los grupos tratados con estos dos formulados, si bien podría deberse a la estimulación de dicha formación, también podría ser consecuencia de una acción inhibidora sobre la colagenasa, lo cual habría permitido, en este caso de heridas abiertas, que se destruyera menos cantidad de colágeno y de esa manera contribuya a la cicatrización.

\section{CONCLUSIONES}

- El champú líquido formulado al 30\% a base del extracto etanólico de Colletia spinosissima presenta efecto cicatrizante en heridas inducidas en piel de ratón.

- El extracto etanólico contiene abundante cantidad saponinas, taninos y flavonoides y presenta actividad antioxidante comparado con el Trolox y vitamina $C$.

\section{REFERENCIAS BIBLIOGRÁFICAS}

1. Hassan KA, Deogratius D, Nyafuono JF, Francis O, Engeu OP. Wound healing potential of the ethanolic extracts of Bidens pilosa and Ocimum suave. African Journal of Pharmacy and Pharmacology 2011; 5(2): 132-6.

2. Süntar IP, Akkol EK, Yilmazer D, Baykal T, Kirmizibekmez $\mathrm{H}$, Alper $\mathrm{M}$, et al. Investigations on the in vivo wound healing potential of Hypericum perforatum L. Journal of Ethnopharmacology 2010; 127(2): 468-77.

3. Prasad V, Dorle KA. Evaluation of ghee based formulation for wound healing activity. Journal of Ethnopharmacology 2006; 107(1): 38-47.
4. Guo S, DiPietro LA. Factors affecting wound healing. J Dent Res 2010; 89(3): 219-29.

5. Brack A. Diccionario enciclopédico de plantas útiles del Perú-Cusco. Centro de Estudios Regionales Andinos "Bartolomé de las Casas". Cusco, 1999. p. 45.

6. Ferreyra R. Sinopsis de la flora peruana. $1^{\text {ra }}$ ed. Editorial Salesiana. Lima, 1979. p. 88.

7. Wang KJ, Li N. Antioxidant phenolic compounds from rhizomes of Curculigo_crassifolia. Arch Pharm Res 2007; 30(1): 8-12.

8. Joseph BS, Justin RS. A comparative study on various properties of five medicinally important plants. International Journal of Pharmacology 2011; 7: 206-11.

9. Lock O. Investigación Fitoquímica. Métodos en el Estudio de Productos Naturales. $2^{\text {da }}$ ed. Fondo Editorial Pontificia Universidad Católica del Perú. Lima, 1994. p. 33-5.

10. Brand-Williams W, Cuvelier ME, Berset C. Use of a free radical method to evaluate antioxidant activity. LWT Food Science and Technology 1995; 28 (1): 25-30.

11. Getie M, Gebre-Mariam T, Rietz R, Neubert RH. Evaluation of the release profiles of flavonoids from topical formulations of the crude extract of the leaves of Dodonea viscosa (Sapindaceae). Pharmazie 2002; 57(5): 320-2.

12. Sriwiroch W, Chungsamarnyart N, Chantakru S, Pongket P, Saengprapaitip K, Pongchairerk U. The effect of Pedilanthus tithymaloides (L.) Poit crude extract on wound healing stimulation in mice. Kasetsart Journal (Natural Science) 2010; 44: 1121-7.

13. Sahasrabudhe A, Deodhar M. Anti-hyaluronidase, antielastase activity of Garcinia indica. International Journal of Botany 2010; 6(3): 299-303.

14. Gurung S, Skalko-Basnet N. Wound healing properties of Carica papaya latex: in vivo evaluation in mice burn model. Journal of Ethnopharmacology 2009; 121(2): 338-41.

15. Ekpo M, Mbagwu H, Jackson C, Eno M. Antimicrobial and wound healing activities of Centrosema pubescens (Leguminosae). Journal of Physics and Chemistry of Solids 2011; 1: 1-6.

Manuscrito recibido el: 07/o8/14

Aceptado para su publicación el: 06/10/14

\section{Correspondencia:}

Nombre: $\quad$ Oscar Herrera Calderón

Dirección: Ciudad Universitaria (Ica, Panamericana Sur Km 305 s/n). Departamento Académico de Ciencias Farmacéuticas. Universidad Nacional San Luis Gonzaga de Ica.

e-mail: $\quad$ oh20oo_4@hotmail.com 